\title{
Rigid bronchoscopy: a general overview
}

\author{
Amit K. Tandon \\ Division of Interventional Pulmonology, Moffitt Cancer Center, Tampa, FL, USA \\ Correspondence to: Amit K. Tandon, MD. 12902 USF Magnolia Drive, Tampa, FL 33612, USA. Email: amit.tandon@moffitt.org.
}

\begin{abstract}
Rigid bronchoscopy developed from an esophagoscope first used by Gustav Killian for the removal of a foreign body. Today rigid bronchoscopes come in a few varieties which differ mainly in their assembly, but the main components are the same. The rigid bronchoscope is used in both therapeutic and diagnostic cases including: tumor excision, stent placement, airway stenosis, and control of hemoptysis. It is imperative to undergo presurgical evaluation by the proceduralist as well as anesthesiologist prior to the procedure which allows discussion of risks and benefits and correction of any reversible contraindication. During the case the type of anesthesia will vary depending on the institution and their comfort level, but the combination of a hypnotic, narcotic and paralytic are the most common combination. After general anesthesia is administered the patient is intubated with the rigid bronchoscope and attached to the ventilator. The most common modes of ventilation are: spontaneous assisted ventilation, positive pressure ventilator and jet ventilation. Reported complications include: damage to the oropharynx, teeth and vocal cords; hemorrhage, hypoxia and laryngospasm. In experienced hands, rigid bronchoscopy is a safe procedure and can offer improved quality of life in those with central airway obstruction.
\end{abstract}

Keywords: Rigid bronchoscopy; endobronchial stent; hemoptysis; central airway obstruction; tracheal stenosis

Received: 09 August 2019; Accepted: 30 October 2019; Published: 10 April 2020.

doi: $10.21037 /$ shc.2019.11.01

View this article at: http://dx.doi.org/10.21037/shc.2019.11.01

\section{Introduction}

The art of bronchoscopy was introduced in the late 1890s by Gustav Killian with the use of a modified esophagoscope (1). With practice on cadavers and continued development, the first foreign body was removed from the airway using a rigid laryngoscope which lead to the era of rigid bronchoscopy. From this foundation, considerable advances have been made to allow the rigid bronchoscope to be used alone and in conjunction with the flexible bronchoscope to complete complex airway procedures.

\section{The rigid bronchoscope}

Many varieties of rigid bronchoscopes exist today with slight differences in the assembly and multifunctional head. The main components however remain the same which include: the barrel, multifunctional head, light source and optics seen on Figures 1 and 2.

The rigid barrel has a beveled tip which is available in two lengths 33 centimeters (tracheal) and 43 centimeters (bronchial) with outer diameters ranging from 6 to $14 \mathrm{~mm}$. The bronchial barrel has fenestrations at the distal end to allow for contralateral ventilation if the barrel is selectively intubated into the right or left mainstem. The tracheal barrel does not include side fenestrations as the length only allows this barrel to reach the mid to distal trachea. The choice depends on the location of the obstruction or lesion as well as goals of the procedure.

The multifunctional head varies in the number of ports available and allows for various instruments to be used during the procedure, including but not limited to: suction catheters, balloons, and biopsy/stent forceps. Connected to this is the anesthesia port comes which is available in two types depending on the mode used for ventilation: jet or volume control.

The light source and optics allow for visualization during the intubation and the procedure. The barrel can be inserted with direct visualization or with the use of a telescope and camera limiting damage to the airway. The 
typical telescope used is $0^{\circ}$ direct visualization and the length is a few centimeters longer than the chosen barrel.

After the airway is secured, the operator can utilize a variety of different instruments depending on the underlying condition and desired procedure. The instruments, when compared to the flexible bronchoscope, are designed to be larger and stiffer, but even with the rigid barrel the flexible scope is used in conjunction in a majority of cases.

\section{Presurgical testing}

During the presurgical evaluation in the office or in the intensive care unit, chest imaging, laboratory values (including, but not limited to: complete blood count, PT/ INR, type and screen, basic metabolic panel), EKG and any other imaging or testing at the discretion of the anesthetist or proceduralist are reviewed.

During the physical exam, special attention should be given to mouth opening, teeth or lack thereof, neck extension and history of prior facial, neck trauma/surgeries, other oral obstructions.

Increased procedural risks occur in those with limited oxygen reserve, poor neck mobility, hemodynamic instability and higher propensity to bleed: inherently or iatrogenically due to medications which should be discontinued if possible.

\section{Contraindications}

Very few contraindications exist for rigid bronchoscopy and, with experienced hands, most of these can be overcome. Relative contraindications include coagulopathy or anticoagulation medication and higher ventilatory requirements. The main absolute contraindication is patients with an unstable cervical spine (e.g., patients with previous radiation, rheumatoid arthritis with neck involvement) risking further damage due to the neck extension which is required for intubation. During the presurgical testing these risks should be addressed with anesthesia and corrected if possible. In all cases, the risk and benefit need to be weighed and discussed with the patient and or power of attorney, as well as, alternative treatments. If the benefit is possible or an alternative is unavailable, then rigid bronchoscopy should be performed.

\section{Anesthesia}

Intubation of the patient requires deep sedation and,

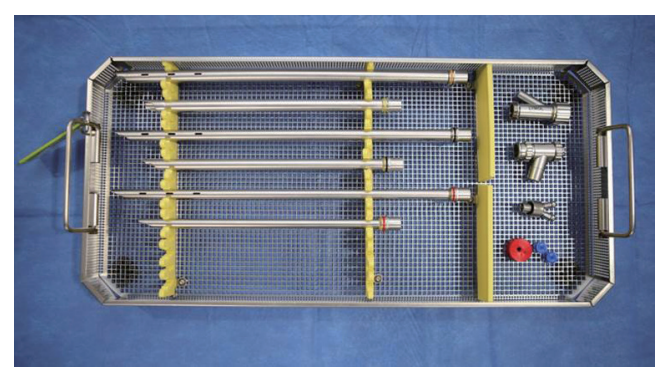

Figure 1 Left-various rigid barrels (tracheal and bronchial); right (top to bottom) - multifunctional head, anesthesia connector, metallic obturator cap for double accessory, silastic caps.

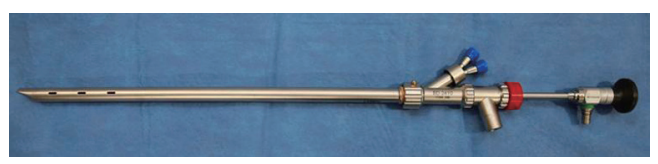

Figure 2 Rigid bronchoscope set up with telescope.

in most instances, paralytic, to prevent injury to teeth, posterior oropharynx, vocal folds and trachea. The rigid bronchoscope needs to be in very experienced hands for those patients in which a paralytic is not utilized.

Medication recommendations include a combination of hypnotic, narcotic and a paralytic. Common induction agents include propofol, ketamine, and etomidate complemented with a narcotic (fentanyl, remifentanil or alfentanil) (2). Remifentanil has been shown to have a faster recovery compared fentanyl in patients during spontaneous assistant ventilation (3). For maintenance, the preferred and most commonly used medication is propofol and the paralytic choice depends on the patient's co-morbid conditions, anesthetist and procedural comfort level, and case length. The common paralytics used include succinylcholine, vecuronium or cisatracurium and rocuronium.

Use of gaseous anesthetics should be avoided due to lack of complete closure of the circuit, as well as, addition and removal of instruments multiple times throughout the case generating larger leaks for short periods of time.

\section{Intubation}

Intubation requires teamwork with anesthesia, procedural nurses, and proceduralist. Patient positioning on the bed is of utmost importance to allow proper neck extension for easier intubation. Utilization of an operating room table 
allows for extension of the neck. The shoulders must be positioned slightly above the breakdown crease to permit proper extension. The anesthetist will then induce the patient and after confirming proper ventilation using bag mask technique a paralytic is given. Next, the neck is extended, a tooth guard is placed on the upper teeth and the scope is handled with the right hand while the mouth is opened with the left. The rigid scope inserted perpendicular to the mouth with the bevel on top just passing the tongue. The index finger acts as a lever as it sits inside the patient's mouth while the thumb rests on the lips/teeth to prevent injury. Upon passing the tongue the scope lowered to near parallel to the patient's chest. Once at the level of the epiglottis, it is lifted up and the vocal cords are visualized. The barrel is then rotated $90^{\circ}$ and the bevel is used to push one of the vocal cords to the side. As the barrel is introduced through the cords, it is again rotated $90^{\circ}$, in the same direction placing the bevel is at the posterior portion of the larynx. The index finger of the left hand is used to maintain lift on the barrel and bevel to prevent injury the posterior membrane while advancing the scope. Once the barrel is at the desired location, usually distal trachea, the ventilator tubing is connected to the anesthesia port of the rigid bronchoscope.

\section{Modes of ventilation}

The particular mode of ventilation will depend on the entire team-their experience and expertise with the different methods, as well as, the available options in the hospital system. Three common techniques are described below.

Spontaneous assisted ventilation is a mode in which intravenous anesthesia is delivered to a level at which the patient is spontaneously breathing. In this technique, paralytic agents are avoided which may prevent post-operative complications such as re-intubation. Complications from this mode of ventilation, in a study by Perrin and colleagues, occurred in about $18 \%$ of patients which included: post-operative hypoxemia, bronchospasm and laryngospasm (4).

On the other hand, if a combination of paralytic or inhaled anesthetic plus intravenous sedation is used, positive pressure ventilation is required. This mode of ventilation is considered controlled ventilation and special attention to prevention of ventilator and inhaled anesthetic leak is necessary. The prevention of leak is overcome by packing the mouth, and using silastic caps on the ends of the rigid bronchoscope ports. The patient's end-tidal $\mathrm{CO}_{2}$ is monitored along with the tidal volumes and adjusted per anesthesia. Inhaled anesthetic is typically avoided due to unavoidable small leaks in the system and exposure to the operating room staff (2).

Jet ventilation uses high pressured gas delivered in short bursts into an open rigid bronchoscope. Given the open system inhaled anesthetic is contraindicated due to large leaks. Monitoring in these patients requires special attention to chest rise and oxygen saturation, but partial pressure of carbon dioxide is monitored only by serial arterial blood gasses or transcutaneous capnographic monitoring.

\section{Indications for rigid bronchoscopy}

The use of rigid bronchoscopy waned after the development of the flexible bronchoscope, but has come back into favor for specific indications which include placement of silicone stents. This article will focus on the therapeutic interventions using the rigid bronchoscope which include tumor excision, stent placement, repair of airway stenosis, and control of hemoptysis.

Airway disease exists in multiple forms which include intrinsic, extrinsic or a combination of both. They are also further differentiated into malignant or benign. Depending on the mixture of the above will guide the proceduralist to the therapeutic options available.

\section{Tumor excision}

Various mechanisms can be used for debulking tumors which are usually used in combination with one another including: mechanical, thermal therapy and cryotherapy.

Mechanical debulking can be accomplished using the beveled end of the rigid barrel in a coring technique, forceps whether rigid or flexible, or a microdebrider. When using the rigid barrel, careful attention must be made to the angle of the beveled edge, amount of pressure used and anatomical location of the obstruction. This type of debulking, typically referred to as apple-coring, can easily cause unwanted trauma including: mucosal tears, hemoptysis, and airway obstruction. As the rigid barrel is advanced, it is also rotated about $90-180^{\circ}$ to allow the bevel to slice through the tumor. Figure 3 shows an example of pre and post apple coring. Simultaneously, lateral pressure is also applied at the stalk to maintain hemostasis as the barrel is advanced. After passing through the obstruction, the large debulking forceps are used to remove the excised tumor all while keeping constant pressure on the wall from 

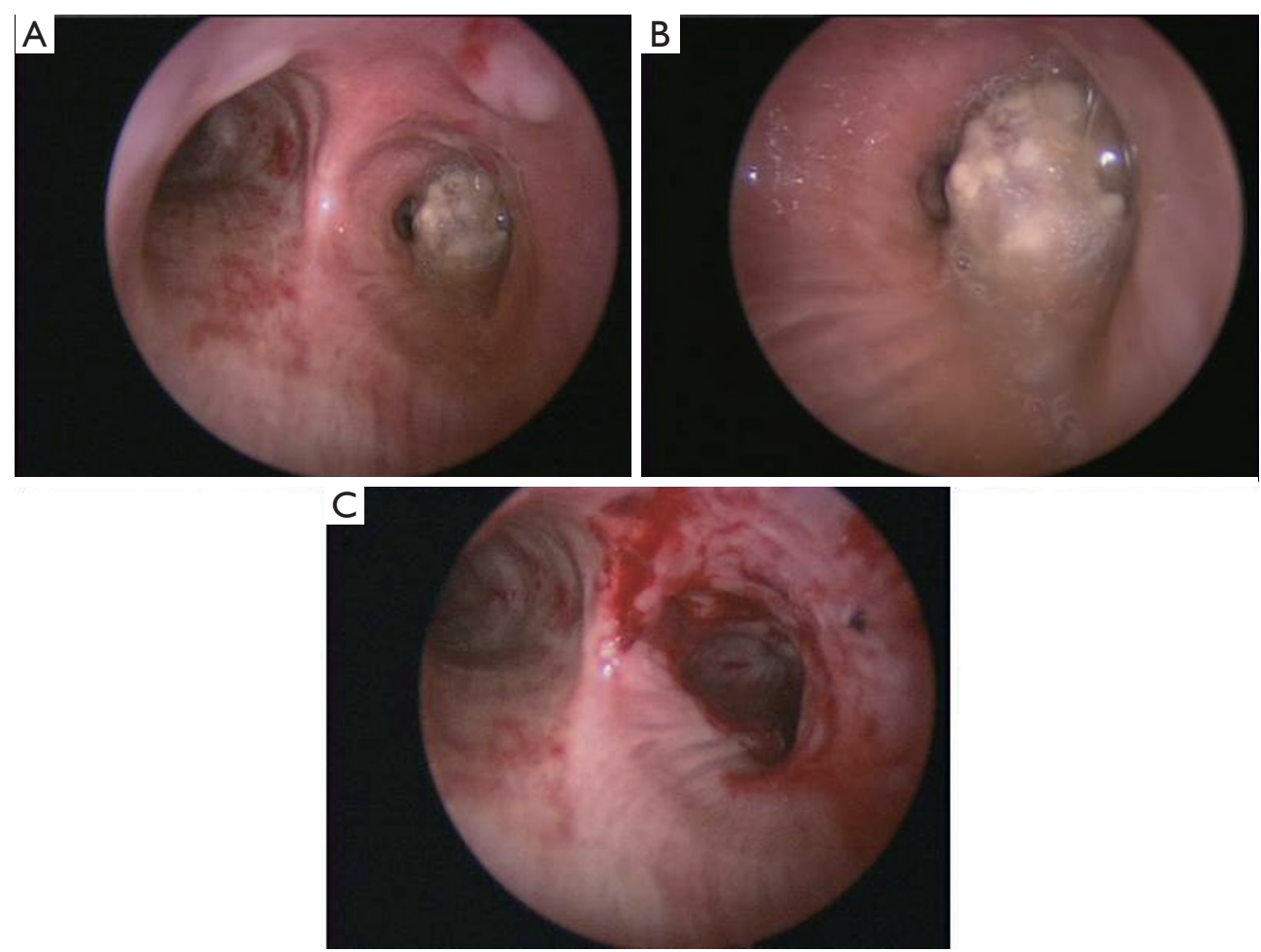

Figure 3 Malignant right mainstem obstruction. (A,B) Tumor arising from the right upper lobe; (C) right mainstem post rigid coring.

which the tumor was excised. Once the tumor is cleared and visualization is restored, the pressure is slowly released to determine if hemoptysis occurs or hemostasis is achieved.

Thermal therapy includes laser (light amplification by stimulated emissions of radiation), electrocautery and argon plasma coagulation (APC). The particular modality chosen is dependent on the proceduralist's experience, location and appearance of the tumor, and depth of penetration desired. The largest limitation of thermal therapy is the required drop in fraction of inspired oxygen $\left(\mathrm{FiO}_{2}\right)$ to less than 0.40 to prevent airway fires.

Laser therapy is best utilized in those tumors which are central, short $(<4 \mathrm{~cm})$ and a visible distal lumen. This is a non-contact modality with the fiber at least 1 centimeter from the tip of the bronchoscope and about 1 centimeter proximal to the lesion (5-9). To achieve carbonization the laser is moved closer to the lesion or multiple pulses are activated (9). Depending on the desired effect the specific laser is chosen for their coagulative or cutting effect. The $\mathrm{CO}_{2}$ and diode lasers are the most precise and the YAG or YAP laser having the best coagulative effect (10). The most common laser used in the endobronchial lesions is the neodymium-yttrium, aluminum, garnet (Nd:YAG) laser
(10). The different properties of lasers depend on their absorption, scatter and depth of penetration.

APC is also a non-contact thermal modality which is utilized more in smaller tumors given the minimal risk for airway perforation. The use of APC or laser can be used prior to mechanical debulking as a method to coagulate and desiccate the tumor allowing for better hemostasis, however this limits the pathologist's review of the specimen due to destruction.

Electrocautery snare allows debulking of tumors with a stalk. During the cutting of the tumor there is electrical current which allows for cauterization and hemostasis. Removal in this fashion allows for preservation of tissue for pathologist review with limited destruction as opposed to mechanical removal with or without prior laser or APC therapy.

Cryotherapy utilizes the Joule-Thompson effect freezing the tissue to $-89^{\circ} \mathrm{C}$ with the use of nitrous oxide. By freezing the tumor, this allows for hemostasis by way of vasoconstriction, platelet aggregation and tumor dehydration. Cryotherapy is also advantageous compared to thermal therapy in those patients in whom limiting $\mathrm{FiO}_{2}$ is not possible. Recommended freeze thaw cycles range from 


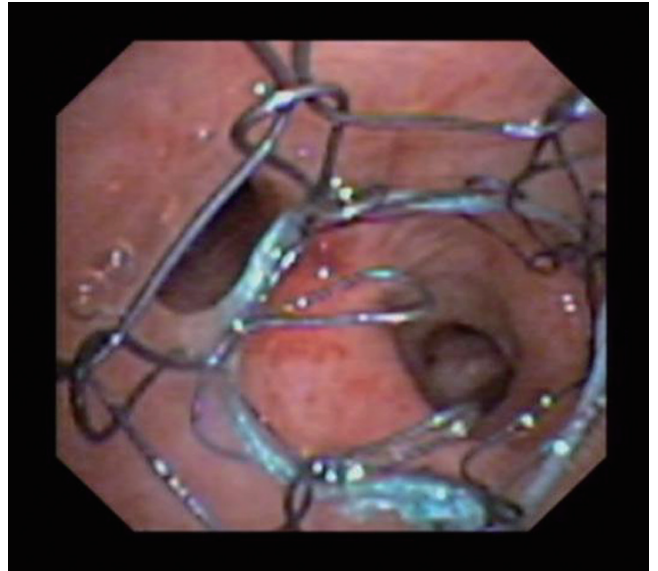

Figure 4 Partially covered metallic stent allowing ventilation of subsegment while covering tumor above.

three to five applications at 30-60 seconds of freezing and then allowing thaw. This method is applied for the entire length of the tumor with overlapping areas. The major downside to cryotherapy for tumor excision is a required bronchoscopy 2-4 days after to remove sloughed tissue.

Ost and colleagues reviewed the benefits of rigid bronchoscopy with a retrospective analysis of the AQUIRE registry. Success in relief of obstruction was obtained in $93 \%$ of patients, improvement in dyspnea in $48 \%$ of patients and improvements in quality of life in $42 \%$ of patients. Those with higher level of baseline dyspnea saw better improvements in dyspnea and quality of life scores and those with lobar obstruction saw fewer improvements (11).

\section{Stents}

Stents come in a variety of diameters and lengths as well as multiple different types of material. The two most common stents are made from metal (Nitonol) with or without a polyurethane cover and silastic. The choice of stent is dependent on the disease as mentioned above.

Metallic stents are typically reserved for malignant airway disease and using covered, partially covered or uncovered is dependent on the location of the disease and whether patency of certain lobes are trying to be maintained.

To select the proper size of the stent a multimodality approach is used. Prior to the procedure the CT scan is used to measure the disease-free airway to give an estimate of what the diseased side should be. At the time of the procedure, estimation of the size can be done with the use of the flexible bronchoscope or rigid barrel (knowing the diameter of either will give an estimate of the size of obstruction) and endobronchial dilation balloons. The initial balloon diameter selected is also guided by the estimated degree of obstruction based on the CT scan measurements, the size compared to the bronchoscope and the normal size of the anatomical airway in healthy patients. The balloon should be at least 5 millimeters longer than the stenosis on either side or serial dilations in an overlapping manner. It is recommended that balloon dilation is done in a serial fashion starting with the smallest balloon size and increase in one millimeter increments. There are no strict guidelines for the balloon inflation time, but for malignant disease we dilated for one minute and for benign disease we dilated for two minutes. If the patient's hemodynamics are intolerant to the dilation at any point the balloon is deflated. After dilation to desired patency, the length of the stent is measured using the bronchoscope. The proceduralist places the bronchoscope at the estimated distal end of the stent and by gripping the bronchoscope at the end of the rigid barrel, the scope is withdrawn to the desired proximal portion of the stent. The length from the end of the rigid barrel to the proceduralist's finger is measured. It is recommended that the stent be at least $5-10 \mathrm{~mm}$ beyond and proximal to the diseased portion of the airway. The diameter of the stent is chosen based on largest balloon dilation and upsizing this by one to two millimeters. Depending on the size of the rigid barrel and location of the stenosis, placement can be done with direct visualization or with the utilization of fluoroscopy with or without a guidewire.

Metallic stents whether covered, uncovered or partially covered are chosen dependent on the location of the disease. If the disease is malignant and spans the entire desired location a completely covered stent is recommended. On the other hand, if the disease is malignant with free portions prior to a secondary carina and patency of the subsegment is trying to be maintained a partially covered stent can be used as seen in Figure 4.

When considering metallic stents for benign airway disease, the United States Food and Drug Administration has placed warnings due to complications in these patients. These stents should be placed and removed in experienced hands due to the high complication risk.

Uncovered stents are typically reserved for airways in which granulation tissue is desired such as in a dehiscence and not recommended in benign airway disease.

Silastic stents are in large part why the rigid bronchoscope came back in favor and are utilized in both benign and 

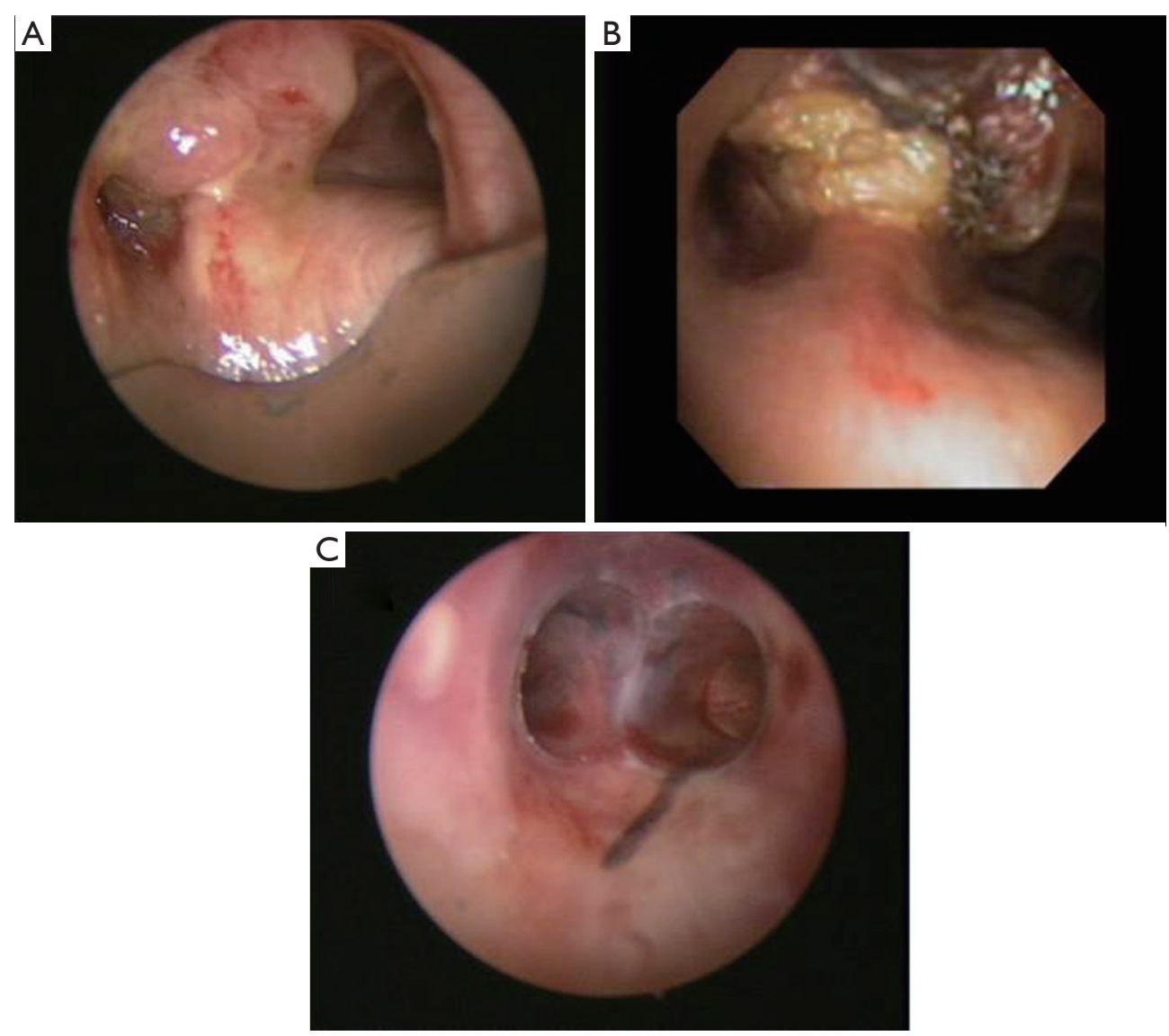

Figure 5 Non-small cell carcinoma involving the main carina. (A) Tumor arising from the main carina and obstructing the right and left mainstems; (B) tumor post laser therapy; (C) post Y stent placement after mechanical debulking and balloon dilation.

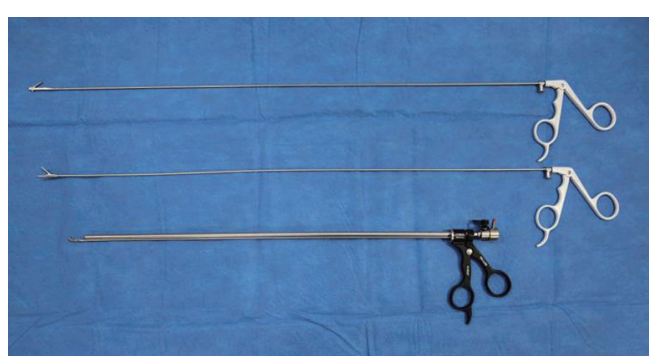

Figure 6 Top to bottom: reverse stent forceps, stent forceps, and large debulking forceps.

malignant disease. Straight stents are reserved for focal areas of stenosis mainly due to benign disease in the trachea or proximal bronchi. Y stents, on the other hand, are reserved for those patients with malignant disease involving the carina and/or distal trachea and are at risk for causing complete airway obstruction as well as benign tracheal stenosis pictured on Figure 5.

For straight stents the rigid bronchoscope is placed slightly distal to the center of the stenosis. As the stent is being deployed the rigid barrel is withdrawn in the opposite direction to ensure correct positioning of the stent. The length of the silastic stents should be at least 1015 millimeters above and below the stenotic area. Once placed, the telescope or the flexible bronchoscope can be used to visualize placement and if needed, the rigid stent forceps can be used to adjust location. Variety of stent forceps are shown in Figure 6.

Measurements for the $\mathrm{Y}$ stent are made in the same fashion as the metallic stents using the flexible bronchoscope and ruler. For the right limb the flexible bronchoscope is flexed to touch the right upper lobe secondary carina and then placed in neutral potion. The scope is then withdrawn to the main carina and this measurement is taken for the distal portion of the right limb. The left mainstem is 


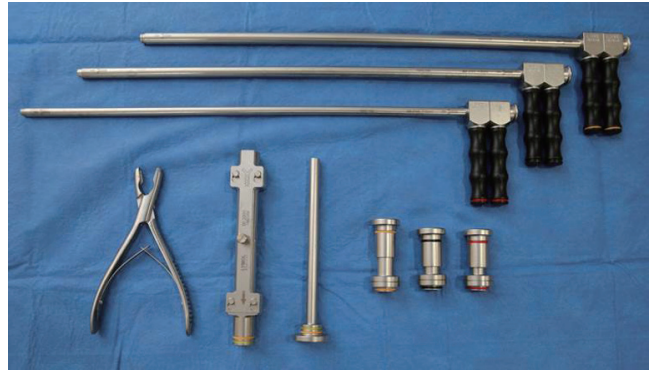

Figure 7 Top-stent deployers, the color/size is matched to the color/size of the barrel. Bottom-(left to right): stent cutter, Y stent loader and plunger, straight stent loaders.

measured to the end of endobronchial disease and typically not longer than 25 to 30 millimeters given the angulation medially of the left mainstem beyond this length. The tracheal length is then measured to the area at least 10 15 millimeters above the disease. When cutting the stent, a scalpel is used with the proceduralist holding either the proximal or distal end of the stent and the assistant holding down the opposite end of the stent to allow for straighter cut. For the right limb, 5 millimeters are subtracted from the measurement of the distal portion measured and the stent is cut at a $45^{\circ}$ angle to match the angle of the right upper lobe in takeoff from the right mainstem. Either the right or left limb is marked in the inside of the stent along with the posterior membrane to allow proper alignment in the deployer prior to inserting this into the rigid barrel. The stent is then loaded in to the $\mathrm{Y}$ stent loader and placed in the stent deployer seen in Figure 7. The rigid bronchoscope is either placed into the left mainstem bronchus or about $20 \mathrm{~mm}$ above the main carina to allow for the bronchial limbs to come out first. Once half way deployed, the rigid bronchoscope is slowly withdrawn with continued deployment of the stent.

The most common stent complications include mucous plugging (4-27\%), granulation tissue (4-10\%) and stent migration (3-10\%) (12-16).

\section{Benign airway stenosis}

Benign airway stenoses occur due to a variety of mechanisms of injury to the native tissue which include: post intubation or tracheostomy, inhalation injuries, infection, or collagen vascular diseases. While surgery is usually the mainstay for complex tracheal stenosis, both simple and complex can be managed bronchoscopically as those presented in Figure 8 .

Simple web-like stenoses can be managed with thermal therapy to create an incision at the site of the web to release the stenosis. After this the airway is dilated to the estimated diameter of the airway determined in a similar fashion as described previously. If possible, the dilation is completed after incisions are made to prevent tears in the mucosa leading to fibroblastic proliferation and further scar tissue. Mitomycin is utilized to help prevent recurrent stenosis as an anti-fibrotic agent with varying success (17-20). It is an antineoplastic antibiotic that acts as an alkylating agent by inhibiting DNA and protein synthesis and with this it can inhibit cell division, protein synthesis, and fibroblast proliferation. Application is done using the rigid or flexible forceps and either gauze or cotton tip applicators soaked in the mitomycin. Using either method the mitomycin solution is held in position for about 1 minute intervals. After each minute a new applicator is grasped with the forceps and applied in a clockwise method at 4-6 points at the site of previous stenosis as presented on Figure 9. More recently Bohra and colleagues have reported success with cryospray and balloon dilation as a safe procedure for tracheal stenosis (21). If there is recurrence either stent or surgical resection should be considered.

Complex airway stenoses which involve the cartilage, stent placement is recommended due to the high recurrence if thermal therapy and balloon dilation are used alone (22-24). Thermal therapy is limited as many of these stenoses have a component of malacia or are damaged cartilage and native wall risking perforation. These stenoses are typically dilated and stent is then placed for months if the patient is not a surgical candidate.

Silastic stents are the stent of choice in benign tracheal stenosis which exist in two forms $\mathrm{Y}$ shaped and straight. Straight stents have a high migration risk and should be considered prior to placement. The patient population for which a $\mathrm{Y}$ stent is used include: distal tracheal stenoses, patients with chronic tracheostomy despite stent placement, and patients at risk for repeated intubations given comorbidities and migration due to manipulation. On the other hand, straight silastic stents are typically used for simple recurrent mid tracheal and bronchial stenosis if surgery is not an option.

\section{Hemoptysis}

Massive hemoptysis is defined differently and ranges from 

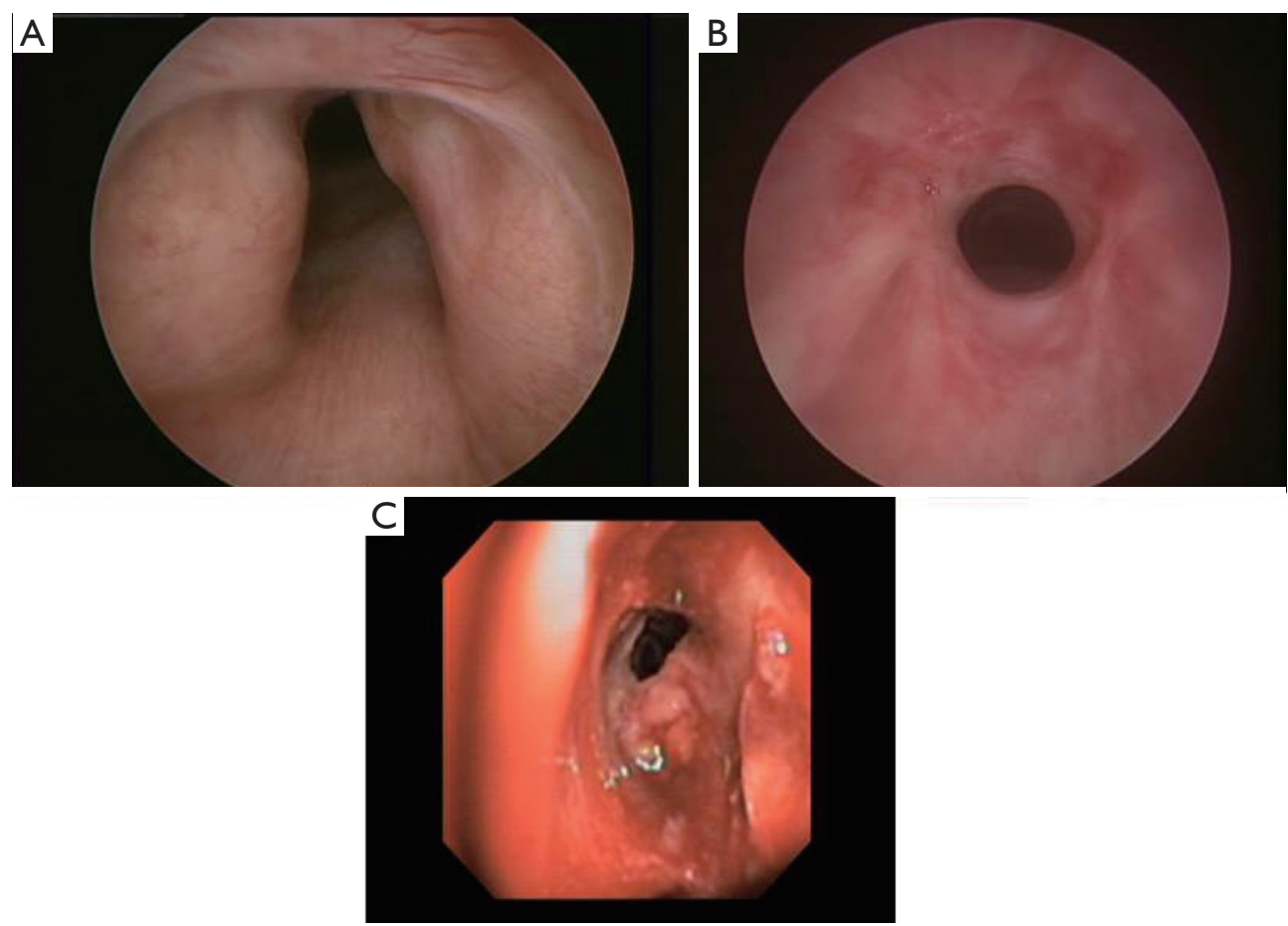

Figure 8 Various forms of tracheal stenosis. (A) Pseudo-glottic tracheal stenosis; (B) simple tracheal stenosis; (C) complex tracheal stenosis.
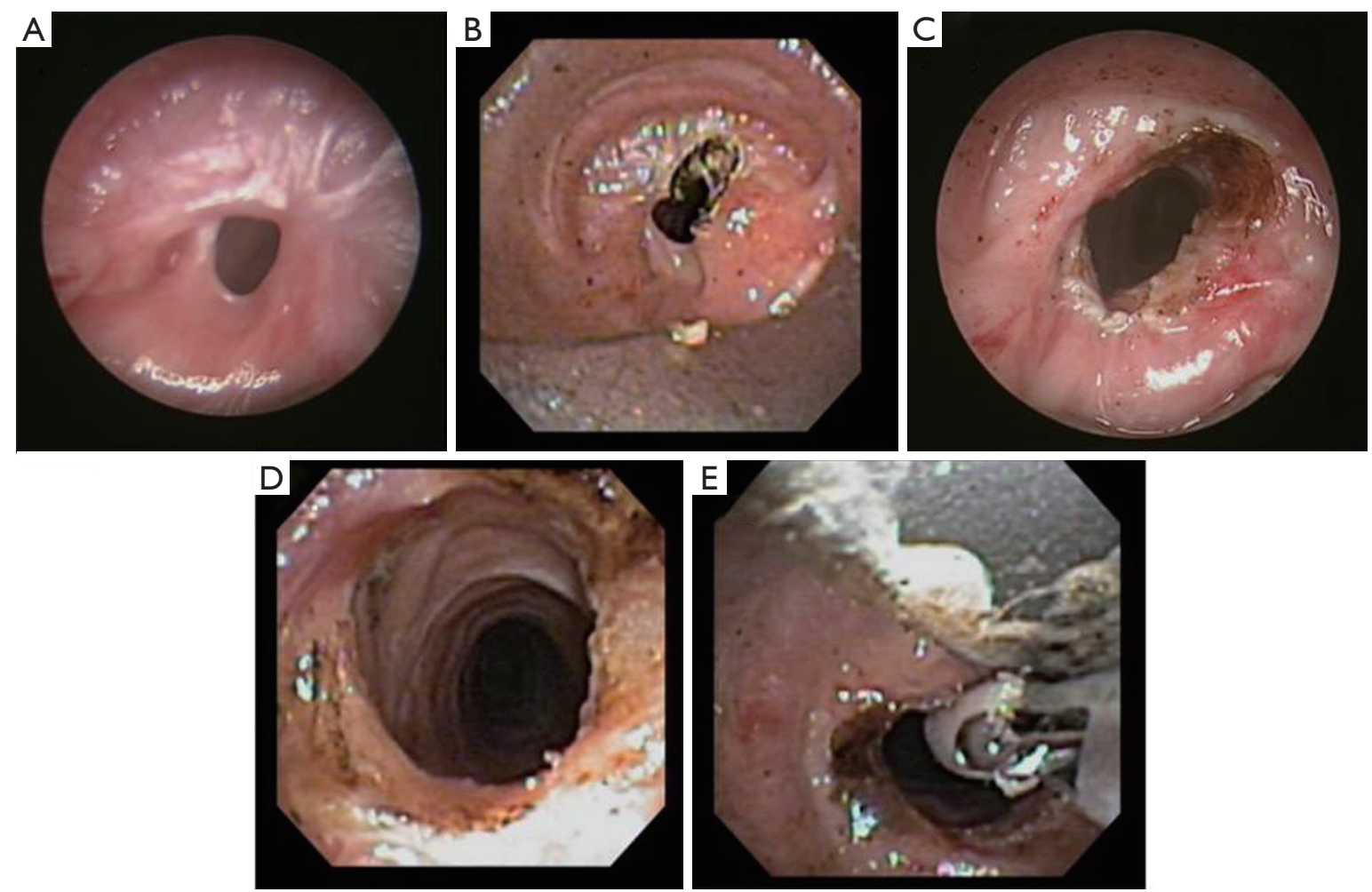

Figure 9 Simple web tracheal stenosis. (A) Tracheal stenosis prior to treatment; (B,C) post holmium: yttrium aluminum garnet laser incision; (D) post balloon dilation; (E) mitomycin-c application with cotton tip applicator. 
100-1,000 mL/24 hours. The rigid bronchoscope allows the proceduralist to secure an airway as well as maintain ventilation. The rigid barrel provides a larger conduit to facilitate easier insertion of instruments to help alleviate ongoing bleeding with various mechanisms: balloon blockers, APC, laser therapy. After bleeding is controlled clot removal can be facilitated with the use of rigid suction, cryoprobe and even rigid forceps.

\section{Complications}

It is hard to determine the exact number of pulmonary physicians who perform rigid bronchoscopies, but the American College of Chest Physicians, European Respiratory Society and American Thoracic Society require 20 such procedures to gain basic competency and thereafter 10 procedures per year to maintain competency $(25,26)$. Given lack of tools to assess true competency, Mahmood and colleagues developed the Rigid Bronchoscopy Tool for Assessment of Skill and Competence to serve as an objective tool of basic rigid bronchoscopy intubation and central airway navigation. The most common mechanical complications of rigid bronchoscopy are trauma to the teeth, oropharynx, vocal cords or other glottic structures, laryngospasm, pneumothorax, and hemorrhage, and death (27-29). Systemic complications include vasovagal syncope, hypoxemia, hypercarbia, medication effects of general anesthesia, arrhythmia, post-procedural respiratory failure, and death $(2,27,28)$.

\section{Conclusions}

The main limitations of the procedure include experience of the operator, requirement of general anesthesia and the sicker population making this at a higher risk procedure. Rigid bronchoscopy is an overall safe procedure for therapeutic and diagnostic interventions while allowing for controlled ventilation. As with any procedure the risks and benefits should be discussed with the patient or power of attorney and if the benefits outweigh the risks, the procedure should not be withheld. Working as a team with the anesthesia staff, nursing and operating room staff, and the proceduralist are imperative to a safe working environment.

\section{Acknowledgments}

Funding: None.

\section{Footnote}

Provenance and Peer Review: This article was commissioned by the Guest Editors (Douglas Kyle Hogarth and Jonathan S. Kurman) for the series "Interventional Pulmonology and Advanced Bronchoscopy" published in Shanghai Chest. The article has undergone external peer review.

Conflicts of Interest: The author has completed the ICMJE uniform disclosure form (available at http://dx.doi. org/10.21037/shc.2019.11.01). The series "Interventional Pulmonology and Advanced Bronchoscopy" was commissioned by the editorial office without any funding or sponsorship. The author has no other conflicts of interest to declare.

Ethical Statement: The author is accountable for all aspects of the work in ensuring that questions related to the accuracy or integrity of any part of the work are appropriately investigated and resolved.

Open Access Statement: This is an Open Access article distributed in accordance with the Creative Commons Attribution-NonCommercial-NoDerivs 4.0 International License (CC BY-NC-ND 4.0), which permits the noncommercial replication and distribution of the article with the strict proviso that no changes or edits are made and the original work is properly cited (including links to both the formal publication through the relevant DOI and the license). See: https://creativecommons.org/licenses/by-nc-nd/4.0/.

\section{References}

1. Killian G. Ueber directe bronchoscopie. MMW 1898;27:844-7.

2. Pathak V, Mahmood W, MacIntyre S. Ventilation and anesthetic approaches for rigid bronchoscopy. Ann Am Thorac Soc 2014;11:628-34.

3. Natalini G, Fassini P, Seramondi V, et al. Remifentanil vs. fentanyl during interventional rigid bronchoscopy under general anaesthesia and spontaneous assisted ventilation. Eur J Anaesthesiol 1999;16:605-9.

4. Perrin G, Colt H, Martin C, et al. Safety of interventional rigid bronchoscopy using intravenous anesthesia and spontaneous assisted ventilation: a prospective study. Chest 1992;102:1526-30.

5. Cavaliere S, Foccoli P, Farina PL. Nd:YAG laser bronchoscopy: a five-year experience with 1,396 
applications in 1,000 patients. Chest 1988; 94:15-21.

6. Kvale PA, Eichenhorn MS, Radke JR, et al. YAG laser photoresection of lesions obstructing the central airways. Chest 1985;87:283-8.

7. Cavaliere S, Venuta F, Foccoli P, et al. Endoscopic treatment of malignant airway obstructions in 2,008 patients. Chest 1996;110:1536-42.

8. Maiwand MO, Mathur PN. Endobronchial cryotherapy. Semin Respir Crit Care Med 1997;18:545-54.

9. Bolliger CT, Sutedja TG, Strausz J, et al. Therapeutic bronchoscopy with immediate effect: laser, electrocautery, argon plasma coagulation and stents. Eur Respir J 2006;27:1258-71.

10. Khemasuwan D, Mehta AC, Wang KP. Past, present, and future of endobronchial laser photoresection. J Thorac Dis 2015;7:S380-8.

11. Ost DE, Ernst A, Grosu HB, et al. AQuIRE bronchoscopy registry. Therapeutic bronchoscopy for malignant central airway obstruction: success rates and impact on dyspnea and quality of life. Chest 2015;147:1282-98.

12. Mudambi L, Miller R, Eapen GA. Malignant central airway obstruction. J Thorac Dis 2017;9 Suppl 10:S1087-110.

13. Wood DE, Liu YH, Vallières E, et al. Airway stenting for malignant and benign tracheobronchial stenosis. Ann Thorac Surg 2003;76:167-72.

14. Lemaire A, Burfeind WR, Toloza E, et al. Outcomes of tracheobronchial stents in patients with malignant airway disease. Ann Thorac Surg 2005;80:434-7.

15. Ost DE, Shah AM, Lei X, et al. Respiratory infections increase the risk of granulation tissue formation following airway stenting in patients with malignant airway obstruction. Chest 2012;141:1473-81.

16. Hohenforst-Schmidt W, Zarogoulidis P, Pitsiou G, et al. Drug Eluting Stents for Malignant Airway Obstruction: A Critical Review of the Literature. J Cancer 2016;7:377-90.

17. Rahbar R, Shapshay SM, Healy, GB. Mitomycin: effects on laryngeal and tracheal stenosis, benefits, and complications. Ann Otol Rhinol Laryngol 2001;110:1-6.

18. Correa AJ, Reinisch L, Sanders DL, et al. Inhibition of subglottic stenosis with mitomycin-C in the canine model.

doi: $10.21037 /$ shc.2019.11.01

Cite this article as: Tandon AK. Rigid bronchoscopy: a general overview. Shanghai Chest 2020;4:15.
Ann Otol Rhinol Laryngol 1999;108:1053-60.

19. Spector JE, Werkhaven JA, Spector NC, et al. Preservation of function and histologic appearance in the injured glottis with topical mitomycin-C. Laryngoscope 1999;109:1125-9.

20. Madan K, Agarwal R, Aggarwal AN, et al. Utility of rigid bronchoscopic dilatation and mitomycin $\mathrm{C}$ application in the management of post intubation tracheal stenosis case series and systematic review of literature. J Bronchology Interv Pulmonol 2012;19:304-10.

21. Bhora FY, Ayub A, Forleiter CM, et al. Treatment of Benign Tracheal Stenosis Using Endoluminal Spray Cryotherapy. JAMA Otolaryngol Head Neck Surg 2016;142:1082-7.

22. Brichet A, Verkindre C, Dupont C, et al. Multidisciplinary approach to management of postintubation tracheal stenosis Eur Respir J 1999;13:888-93.

23. Baugnée PE, Marquette CH, Ramon P, et al. Endoscopic treatment of post-intubation tracheal stenosis. Apropos of 58 cases. Rev Mal Respir 1995;12:585-92.

24. Dalar L, Karasulu L, Abul Y, et al. Bronchoscopic treatment in the management of benign tracheal stenosis: choices for simple and complex tracheal stenosis. Ann Thorac Surg 2016;101:1310-7.

25. Bolliger CT, Mathur PN, Beamis JF, et al. European Respiratory Society/American Thoracic Society. ERS/ATS statement on interventional pulmonology. Eur Respir J 2002;19:356-373.

26. Lamb CR, Feller-Kopman D, Ernst A, et al. An approach to interventional pulmonary fellowship training. Chest 2010;137:195-9.

27. Drummond M, Magalhaes A, Hespanhol V, et al. Rigid bronchoscopy: Complications in a university hospital. J Bronchol 2003;10:177-81.

28. Petrella F, Borri A, Casiraghi M, et al. Operative rigid bronchoscopy: indications, basic techniques and results. Multimed Man Cardiothorac Surg 2014. doi: 10.1093/ mmcts/mmu006.

29. Mahmood K, Wahidi MM, Osann KE, et al. Development of a Tool to Assess Basic Competency in the Performance of Rigid Bronchoscopy. Ann Am Thorac Soc 2016;13:502-11.. 